\title{
Letter
}

\section{A Model for Design Optimization of Electrochemical Mechanical Polishing Polish Pad}

\author{
Bian Yanfei", Wang Zhenxuan, Cai Meng, Wang Ruofu, Tian Jingqun \\ The 54th Research Institute of China Electronics Technology Group Corporation, Shijiazhuang, China \\ Email address: \\ bianyfchengyy@163.com (Bian Yanfei) \\ ${ }^{*}$ Corresponding author

\section{To cite this article:} \\ Bian Yanfei, Wang Zhenxuan, Cai Meng, Wang Ruofu, Tian Jingqun. A Model for Design Optimization of Electrochemical Mechanical \\ Polishing Polish Pad. International Journal of Mechanical Engineering and Applications. Vol. 5, No. 4, 2017, pp. 223-227. \\ doi: 10.11648/j.ijmea.20170504.16
}

Received: July 11, 2017; Accepted: July 19, 2017; Published: August 11, 2017

\begin{abstract}
The polishing pad plays a vital role in achieving the desired removal rate and level of surface planarity during the electrochemical mechanical planarization (ECMP) process. Material removal rate (MRR) and within wafer non-uniformity (WIWNU) are two important factors in determining the polishing performance. In this work, a theoretical model for predicting the radial distribution of tribasic ammonium citrate (TAC) concentration on the wafer is proposed. The experimentally measured MRR was found as a function of the TAC concentration in the slurry. Hence, the model could not only predict the removal rate at a given point on the wafer surface, but also reflect the WIWNU. Model predictions are in good agreement with the experimental data. The proposed model are used to perform an analysis of the effect of pad designs on the MRR and WIWNU of the wafer.
\end{abstract}

Keywords: Electrochemical Mechanical Polishing, Polish Pad, Removal Rate, Within Wafer Non-Uniformity, Model

\section{Introduction}

Chemical mechanical polishing (CMP) technology has become a widely used technique for planarization removal Traditional CMP technology faces severe challenges due to the introduction of new porous low-k dielectric material, which may be damaged easily in the CMP process [1-3]. In order to overcome the inherent defects of CMP, electrochemical mechanical polishing (ECMP) technology has been introduced and gotten more and more attention [4]. The planarization performance of ECMP is significantly influenced by polishing pad characteristics [5-7]. Pad structure and properties are important to determine the removal rate and within wafer nonuniformity (WIWNU) during ECMP process, which are very important factors in determining the polishing performance. The design of the pad is critical in achieving the desirable ECMP performance.

\section{The Model Used to Predict the Removal Rate and WIWNU}

In this work, the slurry delivery system is schematically shown in Figure 1. The pad has an X-Y groove pattern, as shown in Figure 2. Between these grooves, there are pad mesas in the form of squares. The depth and width of the grooves are $h$ and $b$, respectively. The holes are uniformly distributed on the pad surface to supply slurry during the operation. Each hole comes up against a groove junction (black node in Figure 2). The liquid flow rates and directions are determined by the liquid pressure distribution at all the intersections on the pad. The chemicals in the liquid are depleted as the liquid flows away from the injection points on the pad. The chemical reaction continues to occur on the pad groove. At the same time, pad asperities on the pad mesas in contact with the wafer surface mechanically remove the reacted film from the wafer surface. Therefore, the material removal is achieved by chemical dissolution of the copper 
due to chemical reaction and through mechanical removal of a reacted layer formed on the wafer surface by the asperities on the pad mesa.

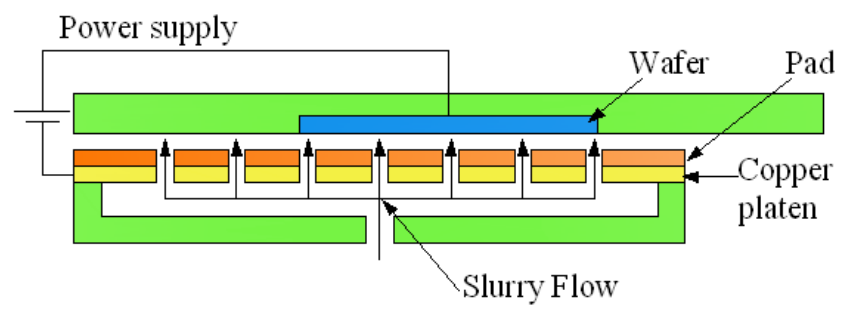

Figure 1. Slurry delivery system in experiments.

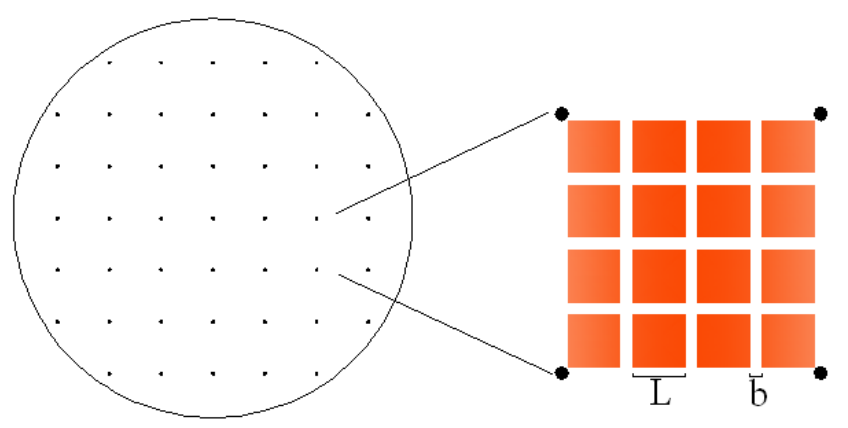

Figure 2. Pad structure.

The basic electrolyte composition in experiment consists of 6 wt.\% hydroxyethylidene diphosphoric acid (HEDP), 0.3 wt.\% 5-Methyl-1H-Benzotriazole (TTA), 3 wt.\% Tribasic Ammonium Citrate (TAC) at pH8. The anodic potential is 2.6 $\mathrm{V}$ (vs. SCE). The copper removal rate on the wafer surface is a function of the TAC concentration on the wafer surface. It is assumed that the TAC concentration only varies with distance along the groove and is uniform at the cross-section. The paper sets the TAC concentration at node $A$ in any groove as $C_{A}$, and the TAC concentration at node $B$ as $C_{B}$. The flow rate of groove is assumed to be $Q_{A B} \mathrm{~m}^{3} / \mathrm{s}$, and the length of the groove is assumed to be $L$. Consider a differential volume of fluid of length $\Delta x$ and occupying the entire cross-section. The area at the top of the groove in contact with the wafer is $2 \mathrm{a} \Delta x$. The rate of copper removal is $2 a \Delta x f(C(x))$. Because two moles of TAC are consumed for every mole of copper removed [8], the rate of TAC consumed in the differential section $\Delta x$ is $4 a \Delta x f(C(x))$. The rate at which TAC enters is $Q_{A B} C(x)$, and that rate at which it leaves is $Q_{A B} C(x+\Delta x)$. Therefore,

$$
Q_{A B}[C(x)-C(x+\Delta x)]=2 \operatorname{na} \Delta x f(C(x))
$$

and in the limit $\Delta x \rightarrow 0$

$$
\frac{d C}{d x}=-\frac{2 n a}{Q_{A B}} f(C(x))
$$

Equation (2) can be integrated from $A$ to $B$ (from 0 to $L$ )

$$
\int_{C_{A}}^{C_{B}} \frac{d C}{f(C)}=-\int_{0}^{L} \frac{2 n a}{Q_{A B}} d x=-\frac{2 n a L}{Q_{A B}}
$$

where $f(C)$ is moles of $\mathrm{Cu}^{2+}$ removed per square meter per second, $C$ is the TAC concentration and has the units of moles of TAC per liter. The result for the removal rate can be converted to $\mathrm{nm} / \mathrm{min}$ by multiplication by a suitable conversion factor. In this work, $f(C)$ can be obtained by fitting the data of copper ECMP experiments, after including the origin point $(0,0)$.

$$
f(C)=-2.124 \times 10^{-7} \cdot C^{2}+3.706 \times 10^{-5} \cdot C
$$

In addition, the unknown parameter $Q_{A B}$ can be obtained as follows. Choosing an arbitrary groove as an example, the relationship between the flow rate in this groove and the pressures at its two ends (node $A$ and node $B$ ) can be written as follows:

$$
Q_{A B}=K_{A B}\left(P_{A}-P_{B}\right)
$$

where $\mathrm{K}_{\mathrm{AB}}$ is a constant for this groove, $\mathrm{P}_{\mathrm{A}}$ and $\mathrm{P}_{\mathrm{B}}$ are the pressures at the beginning and the end of the groove, respectively. The above relationship is a reasonable first approximation for calculating the pressure drop in a pad groove, because the entrance length is small compared with the length of the groove. Assuming steady fully developed laminar Newtonian flow in a typical pad groove, which is modeled as a rectangular duct, the Navier-Stokes equations can be simplified to yield:

$$
\frac{\partial^{2} V_{x}}{\partial y^{2}}+\frac{\partial^{2} V_{x}}{\partial z^{2}}=\frac{1}{\mu} \frac{\partial P}{\partial x}=c
$$

where $\mu$ is the viscosity of the liquid, which is taken to be the viscosity of water at room temperature because of the low chemical concentration in the liquid, $P$ is the dynamic pressure, and $c$ is a constant. At the top and bottom surface and the two side walls, no-slip conditions are imposed. So, the volumetric flow rate can be evaluated by:

$$
Q=\int_{-a}^{a} \int_{-b}^{b} V_{x}(y, z) d y d z=4 \int_{0}^{a} \int_{0}^{b} V_{x}(y, z) d y d z
$$

A mass balance can be written at an intersection of four neighboring grooves, which is defined as node $(i, j)$. The formulation of this mass balance is:

$$
Q_{\text {in }}+q_{\text {sourse }}=Q_{\text {out }}
$$

where $Q_{\text {in }}$ and $Q_{\text {out }}$ are the total volumetric flow rates in those grooves in which the liquid flows into the intersection and those in which the liquid flows out of the intersection, respectively. $q_{\text {sourse }}$ is the flow rate of the fresh liquid if the node is a hole from which fresh liquid comes up and is zero if the node is not a hole.

In addition, the total liquid flow rate $Q_{\text {total }}$ used in the experiment can be expressed as follows:

$$
\sum q_{(i, j)}=Q_{\text {total }}
$$

The assumption that the pressure at the bottom of the holes is the same for all the holes because of the nature of the distribution system for liquid flow. Therefore, the constant $\mathrm{K}$ is assumed to be the same for all the grooves.

It is assumed that the thickness of the wafer is equal to the depth of the recess in the wear ring. The combination of the wafer and the wear ring covers the entire pad surface during polishing. Consequently, the pressure at any node on the pad 
surface and the pressures at its neighboring nodes as well as the liquid flow rates in its neighboring grooves obey the relationship given by Eq. (8).

The liquid is delivered through the holes into the space between the wafer and the pad. The flow rates through different holes are different. The relationship between the flow rate and pressure drop for each hole is given as follows:

$$
Q=K_{1}\left(P_{d}-P_{i, j}\right)=\frac{\pi c^{4}}{8 \mu h}\left(P_{d}-P_{i, j}\right)
$$

where $k_{l}$ is the constant; $P_{d}$ is the pressure of the liquid under the copper plate, which is assumed to be uniform in the following analysis; $P_{i, j}$ is the liquid pressure at the node $(i, j)$; $c$ and $h$ are radius of hole and the depth of copper plate, respectively.

For the hole in the pad designated (i, j), the relationship among the liquid flow rate from the hole $q_{(i, j)}$, the liquid pressure at the node $\mathrm{Pi}, \mathrm{j}$, and the pressure under the copper plate $\mathrm{Pd}$ is given by:

$$
P_{d}-\frac{q_{(i, j)}}{K_{1}}-P_{i, j}=0
$$

Combining Eqs. (8), (9), (10), and (11), the liquid pressure on the pad surface, the liquid flow rates from each hole, and the liquid flow rate in each pad groove can be obtained.

If the TAC concentration at one end of the pad groove is known, the TAC concentration at a distance $x$ from the end is given by:

$$
C(x)=\frac{3.706 \times 10^{-5} C_{A}}{\left(3.706 \times 10^{-5}-2.124 \times 10^{-7} C_{A}\right) e^{1.482 \times 10^{-4} a x / Q_{A B}+2.124 \times 10^{-7} C_{A}}}
$$

The average TAC concentration in a pad groove is given by the integration:

$$
C_{\text {avg }}=\frac{1}{L} \int_{0}^{L} C(x) d x
$$

The calculation of TAC concentrations at all the intersections within the pad is an iterative computational process. The computation begins from the holes, where fresh liquid is supplied and the initial TAC concentration is known. The TAC concentration at an arbitrary in the pad is only determined by the liquid flows entering this intersection, not the liquid flows leaving this intersection.

An important issue in the calculation of the TAC concentration distribution in pad groove system is that the calculation is different for different regions on the pad surface. Due to the relative motion between the wafer and the pad, the pad surface can be divided into two different regions during the polishing process: the area partly covered by the wafer (region I), and the area never covered by the wafer (region II).

In region I, where the wafer does not always cover the pad surface, the chemicals in the liquid have a chance not to react with the wafer surface during a certain portion of each orbit. During the time when the wafer does not cover the pad surface, the chemical concentration in the liquid will maintain its value from one junction to the next. The rest of the time, the TAC in the liquid in each groove react continuously with the wafer surface. The liquid stream from a hole, where fresh liquid is supplied, is considered as starting stream. The chemical concentration in the starting liquid stream will continuously decrease in the flow direction from one junction to the next. Then the incoming streams mix and the concentration of this mixture is the starting concentration for the outgoing streams. The chemical concentration in the outgoing liquid stream continues to decrease until the next junction. Thus the TAC concentration in Region I will be time-dependent and proper modeling of this behavior is too complicated to attempt here. Therefore, the fact that the grooves in Region I come into contact with the wafer only during a fraction of each orbit period is accommodated approximately in this work. This approach should still provide a robust approximation because of the enormous number of orbits executed during even a few seconds. In region II, the liquid may still contain unconsumed chemicals and fresh liquid is supplied from the holes in this area. Thus region II contributes nothing to the material removal.

Consider a point on the pad surface with radius $r$ with respect to the center of functional regions. Assume $\eta$ is the fraction of the time this point is in contact with the wafer surface. If this point is within region II, $\eta=0$. If this point is within region $\mathrm{I}, \eta$ is between 0 and 1 . In this situation, $\eta$ can be calculated by $[9,10]$ :

$$
\eta=\frac{1}{\pi} \arccos \left(\frac{r^{2}+r_{1}^{2}-r_{2}^{2}}{2 r r_{1}}\right)
$$

where $r_{l}$ is the distance between the pad center and the wafer center. $r_{2}$ is the radius of the wafer.

In region $\mathrm{I}$, the TAC concentration is unsteady and changes with time. As noted earlier, it is assumed here that the TAC concentration in region I is also steady, but to evaluate the TAC consumption, the length $L$ of a typical pad groove is replaced by $\eta L$ as a first approximation.

The path of a typical point on the wafer surface with respect to the pad surface nearly completely covers the area of an annulus if given sufficiently long polish time. Therefore, the average TAC concentration in the pad grooves within this annulus and the average TAC concentration of the liquid film on the pad mesas within the annulus are used to evaluate the material removal rate at the selected point on the wafer surface. Numerical calculations were made showing that the average TAC concentration of the liquid on the pad mesas is less than the average TAC concentration in the pad grooves within the path annulus for numerous wafer points at various radial locations. This difference is insignificant compared with the value of the concentration in the grooves. Therefore, it is an excellent approximation to assume that the average TAC concentration on the pad mesas is equal to the average TAC concentration in the pad grooves within the path annulus. 


\section{Experimental Procedure}

All experiments were carried out on an simulated electrochemical mechanical polishing equipment $[11,12]$. The copper removal rate in the experiments is measured by AFM. Seven microindentation were generated on the sample surface by using a G200 Nano Indenter (Agilent Technologies). AFM was used to scan each of them before and after polishing to calculated the actual material removal rate $[13,14]$. The simulated material removal rate can be calculated using the Eq. (4) when the TAC concentration is predicated by the model. The MRR at any site on the wafer surface increase with the consumption of the TAC concentration at this site. The simulated material removal rate and the actual material removal rate measured in experiments on the different radial position of the wafer are displayer in Figure 1 when the number of holes and the radial of the holes are 45 and $0.5 \mathrm{~mm}$, respectively. It is shown that the current theoretical results are in good agreement with the experimental data. The comparison with experimental results will also serve as further validation of the model. It is also indicated that the radial variation of Tribasic Ammonium Citrate (TAC) concentration on the wafer surface can reflect the WIWNU.

\section{Conclusion}

The model can be used as a design tool to optimize the structure of the ECMP polishing pad. Figures 4, and 5 illustrate the effect of the number and radial of the hole on the distribution of the TAC concentration, respectively. Figure 4 indicates that there are the higher MRR and lower WIWNU when the hole is 45 . Figure 5 indicates that the MRR decrease with the increase of the radial of hole, but WIWNU increase with the radial of hole. The modeling results should be insightful to polishing pad developers to design the next generation of polishing pads.

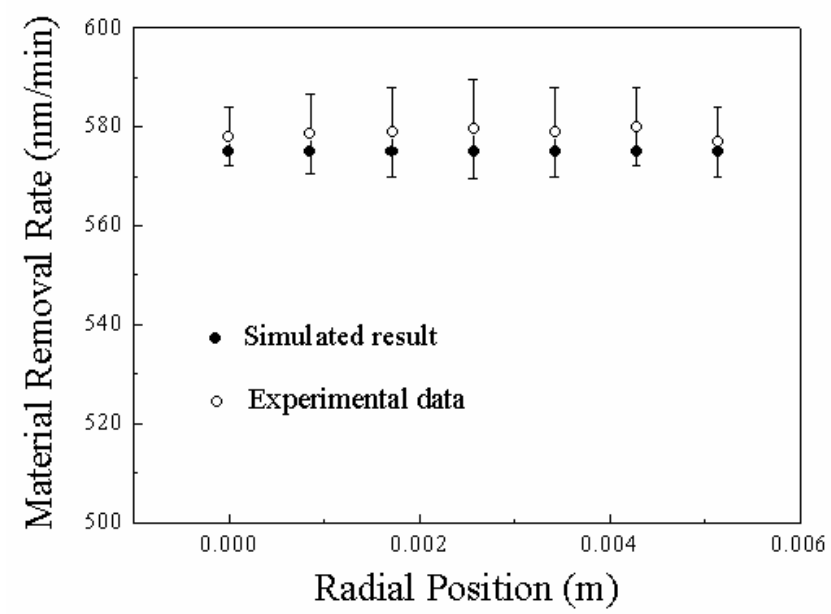

Figure 3. Comparison between the MRR predicated by the model and the $M R R$ measured in experiments at different radial position on the wafer.

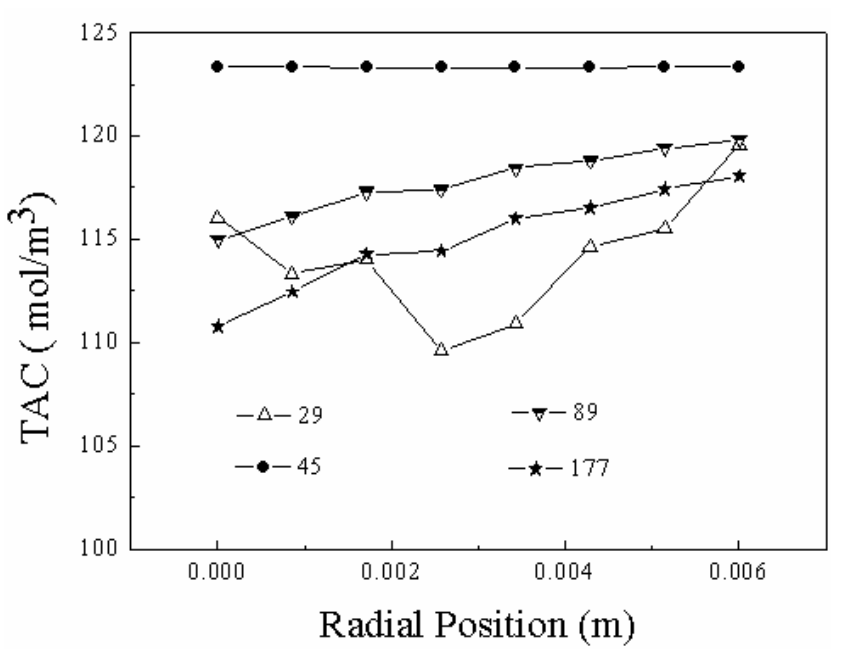

Figure 4. The radial variation of theoretical TAC concentration with different number of holes.

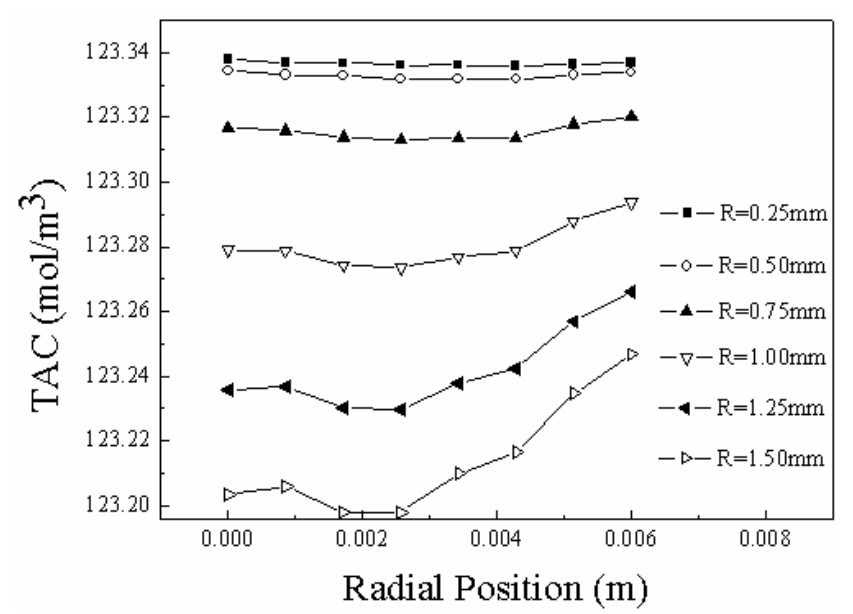

Figure 5. The radial variation of theoretical TAC concentration with different $R$.

\section{References}

[1] K. Mahadevaiyer, J. W. Nalaskowski and L. M. Cook: Chem. Rev. Vol. 110 (2010), p 178.

[2] J. Y. Lin, S. W. Chou: Electrochimica Acta. Vol. 56 (2011), p 3303.

[3] L. Zhang, B. G. Zhang, B. C. Pan and C. W. Wang: Applied Surface Science. Vol. 422 (2017), p 247.

[4] F. Q. Liu, T. Du, A. Duboust, S. Tsai and W. Hsu: J. Electrochem. Soc. Vol. 153 (2006), p 377.

[5] F. Gao, H. Liang: Journal of Electronic Materials. Vol.41 (2013), p 624.

[6] G. S. Kristin, C. W. Alan: Journal of Applied Electrochemistry. Vol. 39 (2009), p 1719.

[7] J. J. Murata, K. S. Yodogawa and K. Ban: INT J MACH TOOL MANU. Vol. 114 (2017), p 1.

[8] Y. J. Oh, G. S. Park and C. H. Chung: J ELECTROCHEM SOC. Vol. 153 (2006): G 617. 
[9] H. Kim and H. Jeong: J ELECTRON MATER. Vol. 33 (2004), p 53.

[10] F. Tyan: IEEE T SEMICONDUCT M. Vol. 20 (2007), p 451.

[11] Y. F. Bian, W. J. Zhai and Y. Y. Cheng: J CENT SOUTH UNIV T. Vol. 21 (2014), p 2191.
[12] Y. F. Bian, W. J. Zhai and Y. Y. Cheng: Trans. Nonferrous Met. Soc. China. Vol. 23 (2013), p 2431.

[13] Y. F. Bian, W. J. Zhai and Y. Y. Cheng: J CENT SOUTH UNIV T. Vol. 21 (2014), p 4157.

[14] F. Gao, H. Liang: Journal of Electronic Materials. Vol. 40 (2011), p 134. 